\title{
Die Entwicklung einer Ansaat auf einem Grundwasserstandsversuch in der Boker Heide
}

\section{P. BOEKER}

Institut für Pflanzenbau der Rheinischen Friedrich-Wilhelms-Universität, Bonn, Bundesrepublik Deutschland

\section{Zusammenfassung}

Auf dem Grundwasserstandsversuch in der Boker Heide ergaben sich vier Jahre nach der Ansaat bei ausschliesslicher Wiesennutzung bei den yerschieden hohen Grundwasserständen sehr deutliche Unterschiede in der Zusammensetzung der Pflanzenbestände, die jetzt schon jeweils zu bestimmten Pflanzengesellschaften zugeordnet werden konnten, obwohl die Entwicklung noch nicht völlig abgeschlossen ist.

Diese Pflanzengesellschaften sind gute Anzeiger für den Feuchtegrad des Bodens, desgleichen auch für dessen Nährstoff- und Reaktionszustand.

\section{Summary}

The development of newly sown grassland on a trial field with different ground-water levels on the Boker Heide

The ground-water level trial field with varying ground-water levels on the Boker Heide was used solely as meadow. Four years after it had been sown, very striking differences were found in the sward composition of plots. These differences can already be attributed to specific plant communities, although development is still incomplete.

These plant communities are good indicators of the degree of humidity of the soil and also of the nutrient status and the degree of alkalinity.

\section{Einleitung}

Uber den Einfluss verschieden hohen Grundwassers auf die botanische Zusammensetzung von Grasland auf schweren Tonböden berichtete MinderHOud (1960), dass dieser relativ gering war. In Ergänzung hierzu soll von den Beobachtungen auf einem Grundwasserstandsversuch in der Boker Heide berichtet werden.

\section{Methode und Material}

Der Versuch liegt im östlichen Westfalen, nordwestlich Paderborn; er wurde 1959 angelegt; Einzelheiten hinsichtlich der Versuchsanlage bringt eine frühere Arbeit (BoEKER u. Кмосн, 1963). Der Versuch liegt auf sehr leichten Sandböden, die ehemals stark sauere Heidepodsole waren, durch die nun fast hundert Jahre andauernde Bewässerung mit kalkreichem Wasser heute aber pH-Werte von über 7,0 (in $\mathrm{KCl}$ ) aufweisen.

Um die Einstellung bestimmter Grundwasserstände zu gewährleisten, wurden die vorhandenen Wässerbeete eingeebnet. Nach verschieden starkem Aushub des aus gelbem

Zur Veröffentlichung erhalten am 30. Dezember 1964. 
Sand bestehenden Untergrundes wurden über einer mit Hilfe eines Grabensystems geschaffenen einheitlichen Grundwasseroberfläche durch Wiederauftrag der Krume Terrassen geschaffen, die $30,50,70,90$ und $150 \mathrm{~cm}$ darüber liegen.

Bei den Bauarbeiten wurde die alte Grasnarbe durch das Abtragen und Wiederaufbringen der Krumenschicht völlig zerstört, so dass eine Neuansaat erforderlich wurde. Hierzu wurde die folgende Mischung benutzt, die auf Vorschläge der für dieses Gebiet zuständigen landwirtschaftlichen Beratungsstelle zurückging:

$\begin{aligned} 20 \mathrm{~kg} / \mathrm{ha} \text { Festuca pratensis } & 3 \mathrm{~kg} / \mathrm{ha} \text { Trifolium repens } \\ 6 \mathrm{~kg} / \mathrm{ha} \text { Lolium perenne } & 1 \mathrm{~kg} / \mathrm{ha} \text { Trifolium pratense } \\ 6 \mathrm{~kg} / \mathrm{ha} \text { Phleum pratense } & 1 \mathrm{~kg} / \mathrm{ha} \text { Lotus corniculatus } \\ 3 \mathrm{~kg} / \mathrm{ha} \text { Poa pratensis } & \end{aligned}$

Die Saatmenge war mit $40 \mathrm{~kg} / \mathrm{ha}$ relativ hoch, sie stellte eine Sicherung für das Gelingen auf den stark durch die Bauarbeiten veränderten Böden dar. Die Vielseitigkeit in der Artenzusammensetzung sollte auf die für später geplante getrennte Nutzung der Flächen als Wiese und als Weide Rücksicht nehmen.

Auf den Parzellen mit den Grundwasserständen von 70, 90 und $150 \mathrm{~cm}$ erfolgte die Ansaat im Frühjahr 1959, auf denjenigen mit 30 und $50 \mathrm{~cm}$ erst im Herbst 1959. Diese Verzögerung ergab sich dadurch, dass es bei den Bauarbeiten bis dahin noch nicht gelungen war, den Einfluss eines benachbarten, höher gelegenen Zuwässerungsgrabens auszuschalten. Dieser bewirkte durch austretendes Druckwasser während der Zeit der Frühjahrsbewässerung der benachbarten Wiesen eine starke Vernässung der tiefergelegenen Parzellen, so dass eine gleichzeitige Ansaat aller Versuchsparzellen unmöglich war. Hierdurch ergaben sich leider zunächst gewisse Auswirkungen auf die Entwicklung der Pflanzenbestände und auch auf die Erträge, die mehrere Jahre anhielten. Sie hatten ihre Ursache vor allem darin, dass nach der Ansaat eine langanhaltende Trockenzeit einsetzte; von April bis Juni 1959 fielen nur 46,3\% des langjährigen Mittels als Niederschlag, von Juli bis September sogar nur 31,7\% und von Oktober bis Dezember 48,3\%. Besonders die Parzellen mit dem Grundwasserstand von $150 \mathrm{~cm}$ litten sehr stark unter der Dürre, hier konnte im ganzen Jahr nur ein Schnitt genommen werden, während die Parzellen mit den Grundwasserständen von 90 und $70 \mathrm{~cm}$ zwei brachten. Das Ausbrennen der Flächen führte natürlich zur Bildung von Lücken, in denen sich dann andere Pflanzen ansiedeln konnten. Besonders auffällig ist seitdem das starke Auftreten von Holcus lanatus, Plantago lanceolata und Rumex acetosa, aber auch von eine Reihe anderer Arten.

\section{Ergebnisse}

In-den folgenden Jahren entwickelten sich dann die Pflanzenbestände ohne Besonderheiten gut weiter. Die angesäten Arten machten den grössten Anteil am Bestand aus. Bei den hohen Grundwasserständen von 30,50 und $70 \mathrm{~cm}$ betrug der Anteil von Festuca pratensis und Phleum pratense über $50 \%$ des Aufwuchses, bei denen von 90 und $150 \mathrm{~cm}$ stieg der Anteil von Lolium perenne stark an. Die nicht angesäten Arten hatten bis 1961 nur einen Anteil von 10 bis $20 \%$ des Bestandes erreicht.

Bis zum ersten Schnitt im Frühjahr 1961 wurde der Versuch einheitlich als Wiese genutzt, danach wurde er zur Hälfte in Weidenutzung genommen. Diese wirkt sich naturgemäss anders auf die Bestandsentwicklung aus als die Wiesennutzung. Da die Einwirkungszeit der Weide noch relativ kurz ist und die Umwandlung der Pflanzenbestände auf den Weideparzellen noch nicht abgeschlossen ist, wird im folgenden nur über die Entwicklung auf dem Wiesenteil des Versuchs berichtet. 
Der Winter 1962/63 brachte eine sehr lange Frostperiode, die Ende Dezember begann und viele Wochen mit sehr tiefen Temperaturen anhielt. Das hatte auch deutliche Auswirkungen auf die Pflanzenbestände. Starke Frostschäden traten besonders bei den mittleren Grundwasserständen von 70 bis $90 \mathrm{~cm}$ Tiefe auf. Wegen des milden Wetters im Vorwinter waren auf diesen Grundwasserstufen hier besonders die Gräser noch gut weitergewachsen, sie wurden dann offensichtlich vom Frost in einem besonders empfindlichen Wuchsstadium stark geschädigt. Das betraf vor allem Lolium perenne, dass in solchen Wintern, wie schon DE VRIES (1942) nachwies, immer erheblich zurückgedrängt wird. Auf den Parzellen, auf denen zum Herbst hin noch stark mit Stickstoff gedüngt worden war, waren sogar fast alle angesäten Gräser verschwunden. Der Aufwuchs auf ihnen bestand dann im Frühjahr 1963 fast nur noch aus Rumex acetosa und Rumex crispus mit wenig Holcus lanatus. Auf den Grundwasserstufen von $150 \mathrm{~cm}$ und denen von 30 und $50 \mathrm{~cm}$ blieben solch starke Schäden aus. Hier unterblieb anscheinend die herbstliche Wachstumsanregung, weil es dort $\mathrm{zu}$ trocken, bezw. zu nass und kalt gewesen war.

Nachdem die Winterschäden sich etwas ausgeglichen hatten, wurden auf dem Versuch und einer angrenzenden alten Wiese im Juni 1963 genaue Bestandsuntersuchungen durchgeführt, deren Ergebnisse die TABELLE wiedergibt.

TABELLe. Botanische Zusammensetzung der Grasnarbe (Methode Klapp-Stählin) auf den verschiedenen Grundwasserstufen am 12.6.1963 und einer benachbarten alten Wiese

Botanical composition of the sward (Klapp-Stählin Method) at different ground-water levels and on an adjacent old meadow on 12.6.1963

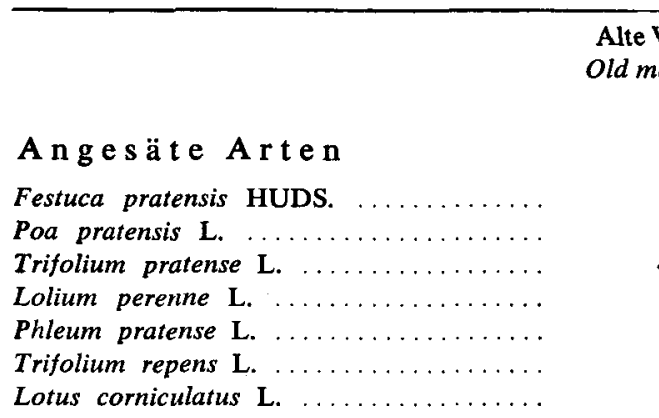

Kennarten der Glatthaferwiesen (Arrhenatheretalia C.A.)

Bellis perennis L.

Chrysanthemum leucanthemum L. .....

Dactylis glomerata L. .............

Anthriscus silvestris HOFFM.

Crepis biennis L. ..............

Galium mollugo L. ...............

Heracleum sphondylium L. ...........

Arrhenatherum elatius J. et PRESL. ....

Pastinaca sativa L. . . . . . . . . . .

Pimpinella magna L. . . . . . . . .

Bromus mollis L. ................

Trisetum flavescens P.B.

Trifolium dubium SIBTH.

+
6
+
2
2
6
2
12
+
+
+
5
+

\begin{tabular}{ccccc}
\multicolumn{5}{c}{$\begin{array}{c}\text { Grundwasserstand in } \mathrm{cm} \\
\text { Water table in } \mathrm{cm}\end{array}$} \\
\hline 150 & 90 & 70 & 50 & 30
\end{tabular}

2

3
4

$\begin{array}{rrrrr}30 & 15 & 30 & 50 & 35 \\ 8 & 5 & 3 & 7 & 8 \\ 2 & 2 & 3 & 1 & 1 \\ 2 & 2 & + & 2 & 3 \\ 10 & 8 & 8 & 8 & 10 \\ + & + & + & + & + \\ + & + & 1 & + & \end{array}$

$\begin{array}{rrrrr}1 & + & + & + & + \\ 3 & 3 & 5 & + & + \\ 1 & + & + & & + \\ + & + & + & & \\ 1 & 1 & + & & \\ 3 & 1 & + & & \\ + & + & + & & \\ 1 & + & & \end{array}$

Fortsetzung S. 4 


\begin{tabular}{cccccc} 
Alte Wiese & \multicolumn{4}{c}{$\begin{array}{c}\text { Grundwasserstand in } \mathrm{cm} \\
\text { Old meadow }\end{array}$} & \multicolumn{4}{c}{ Water table in $\mathrm{cm}$} \\
\cline { 2 - 6 } & 150 & 90 & 70 & 50 & 30
\end{tabular}

Kennarten der Feuchtwiesen (Molinietalia-C.A.)

Lychnis flos cuculi $\mathbf{L}$................ +

Cirsium oleraceum SCOP.

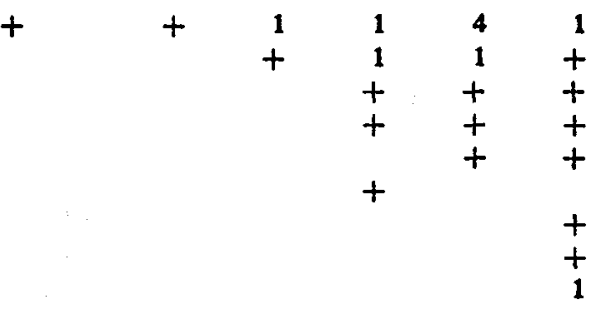

Phalaris arundinacea $\mathrm{L} . \ldots \ldots \ldots \ldots$

Angelica silvestris $\mathrm{L} . \ldots \ldots \ldots \ldots \ldots$

Senecio aquaticus HUDS.

Filipendula ulmaria MAXIM.

Caltha palustris $\mathrm{L} . \ldots \ldots \ldots \ldots \ldots \ldots$

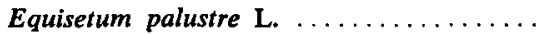

Juncus lamprocarpus EHRH.

Kennarten des Wirtschaftsgrülandes

(Arrhenatherete a-C.A.)

Festuca rubra $\mathrm{L}$.

15
5
2
1
5
2
4
5
+
6

Holcus lanatus L. . . . . . . . . . .

Poa trivialis L.

Lathyrus pratensis $\mathbf{L}$.

Centaurea jacea $\mathrm{L}$.

Plantago lanceolata $\mathrm{L}$.

Ranunculus acer L.

Rumex acetosa $\mathbf{L}$.

Vicia cracca $\mathrm{L}$.

Alopecurus pratensis $\mathrm{L}$

Cerastium caespitosum GIL.

Cardamine pratensis $\mathbf{L}$.

2
8
2
1
2
1
10
+

$\begin{array}{rrrr}3 & 1 & + & + \\ 20 & 15 & 2 & 2 \\ 3 & 2 & 3 & 7 \\ + & + & + & + \\ 2 & 3 & 1 & + \\ 12 & 10 & + & + \\ 3 & 4 & 3 & 5 \\ 15 & 4 & + & + \\ + & + & + & \\ + & + & + & + \\ + & & & +\end{array}$

\section{B e gle it e r}

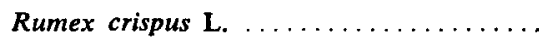

Ranunculus repens $\mathrm{L}$.

Anthoxanthum odoratum $\mathbf{L}$.

Taraxacum officinale L

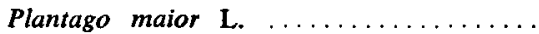

Symphytum officinale $\mathbf{L} . \ldots \ldots \ldots \ldots \ldots$

Glechoma hederaceum L.

Rorripa silvestris (L.) BESS.

Poa annua $\mathbf{L}$.

Leontodon autumnalis $\mathrm{L} . \ldots \ldots \ldots \ldots$

Agrostis alba $\mathbf{L}$.

Glyceria fluitans R. BR. . . . . . . .

Rhinanthus minor L. . . . . . . . .

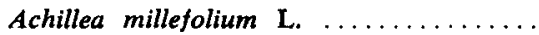

Ajuga reptans L. .................

Arctium lappa L. ...............

Trifolium hybridum L.

$\begin{array}{rrrrrr}+ & 1 & 1 & 1 & + & + \\ 1 & & 1 & 6 & 15 & 20 \\ 10 & 2 & 3 & 2 & & \\ + & 1 & + & + & & \\ & & + & + & + & + \\ & + & + & + & + & + \\ & + & + & + & & + \\ & + & + & & & + \\ & & + & + & & + \\ & & & & 3 & + \\ & & & & + & + \\ & & & & & +\end{array}$

$t=$ spurenweise, unter $1 \%$ des Aufwuchses

Die TABelle gibt die nach der Methode Klapp-Stählin (KLAPP, 1949) geschätzten Ertragsanteile des Aufwuchses wieder; + bedeutet spurenweises Auftreten, d.h. An-

Neth. J. agric. Sci., Vol. 13 (1965) No. 2 (June) 
teil unter $1 \%$. Sie wurde nach pflanzensoziologischen Gesichtspunkten geordnet unter Benutzung des Systems von Braun-Blanquet und Tüxen, da sich hiermit besonders gut der Einfluss der Höhe des Grundwasserstandes auf das Auftreten der einzelnen Arten aufzeigen liess. Es war ein wenig überraschend, dass sich schon vier Jahre nach der Ansaat so deutliche Differenzierungen nachweisen liessen. Ausser den angesäten 7 Arten waren bis zu dieser Zeit schon 47 weitere Arten zusätzlich aufgetreten, zum Teil mit bedeutenderen Mengenanteilen. Das ist auf verschiedene Faktoren zurückzuführen.

Wie schon ausgeführt, litten die Versuchsflächen 1959 unter Trockenheit, 1962/63 durch Frost, so dass Lücken auftraten, in denen sich andere Arten als die ausgesäten ausbreiten konnten. Ferner begünstigte die Wiesennutzung u.a. durch die längeren Intervalle zwischen den einzelnen Nutzungen das Aufkommen vieler Arten. Wiesen sind daher immer artenreicher als Weiden, deren Aufwuchs durch den ständigen Verbiss und Tritt der Tiere sehr viel artenärmer wird. Das lässt sich auch bei den botanischen Untersuchungen auf dem Weideteil des Versuchs in der Boker Heide nachweisen. Die neu aufgetretenen Arten stammen zum grossen Teil aus im Boden befindlich gewesenen Samen der ehemals dort vorhandenen Wiesen, die in ihrer Bestandszusammensetzung der in der TABELLE aufgeführten etwa geglichen haben. Ferner wird es sich bei einzelnen Arten auch um den Neuaustrieb aus alten Rhizomen und Wurzelstöcken handeln. Das ist sicher zum Teil der Fall bei Anthriscus silvestris, Equisetum palustre, Heracleum sphondylium, Rumex acetosa, Symphytum officinale und anderen ähnlichen Arten. Zum Teil werden Samen auch bei der Bearbeitung und Beerntung durch die Geräte oder mit der Stallmistdüngung auf die Flächen gekommen sein (BoEkER, 1959; Korsmo, 1930). Ein weiterer Teil der Arten stammt auch wohl aus dem Samenzuflug von den benachbarten Grünlandflächen, die überwiegend als Wiese genutzt werden únd sehr artenreich sind, weil ihre Nutzung bisher nicht sehr intensiv ist.

\section{Diskussion}

Von den angesäten Arten haben sich nur Festuca pratensis, Phleum pratense und Poa pratensis etwa der Vorausberechnung entsprechend entwickelt. Auffällig ist nur der geringe Anteil von Festuca pratensis bei $90 \mathrm{~cm}$ Grundwasserstand, was vielleicht auf den Frostschäden beruht. Alle anderen angesäten Arten sind nur noch in sehr geringen Anteilen vertreten, zum grossen Teil sind sie durch die höherwachsenden Arten unterdruickt worden. Zwischen den Parzellen mit den verschieden hohen Grundwasserständen lässt sich mit ihrer Hilfe jedoch keine Aussage über deren Auswirkung auf die Bestandesentwicklung machen. Das ist erst möglich, wenn man dazu die inzwischen aufgetretenen anderen Arten mit berücksichtigt. Die Entwicklung der Pflanzenbestände war im Juni 1963 sicherlich noch nicht ganz abgeschlossen. Der Grundstock der Arten der nach der vorliegenden Höhe der Grundwasserstände zu erwartenden Pflanzengesellschaften war bis dahin aber schon aufgetreten; in Zukunft sind nur noch relativ geringfügige Ergänzungen $\mathrm{zu}$ erwarten.

Unter Zugrundelegung des Systems Braun-Blanquet sind in enger Anlehnung an TüxEN und Preising (1951) die vorgefundenen Pflanzenbestände folgenden Pflanzengesellschaften zuzuordnen:

a. bei $150 \mathrm{~cm}$ Grundwasserstand: Arrhenatheretum typicum - Reine Glatthaferwiese 
b. bei $90 \mathrm{~cm}$ Grundwasserstand: Arrhenatheretum, Subass. von Cirsium oleraceum

- feuchte Glatthaferwiese

c. bei $70 \mathrm{~cm}$ Grundwasserstand: Cirsium oleraceum-Polygonum bistorta-Ass., Subass. von Heracleum sphondylium - BärenklauKohldistel-Wiese

d. bei $50 \mathrm{~cm}$ Grundwasserstand: Cirsium oléraceum-Polygonum bistorta-Ass., typi-

e. bei $30 \mathrm{~cm}$ Grundwasserstand: Cirsium oleraceum-Polygonum bistorta-Ass., Subass. von Carex fusca - nasse Kohldistel-Wiese

Neben der Anzeige für einen bestimmten Feuchtezustand des Bodens lässt sich aus der Art der genannten Pflanzengesellschaften auch ableiten, dass es sich bei deren Standorten um solche mit zumindest befriedigenden Reaktionsverhältnissen und guter Nährstoffversorgung handelt. Glatthaferwiesen und Kohldistelwiesen gedeihen in dieser Zusammensetzung nur auf reicheren Böden. Ausgesprochen säureholde Arten fehlen, dafür sind Arten mit vorwiegendem Auftreten im schwach saurem und neutralen Reaktionsbereich stark vertreten (BoEKER, 1964).

Wenn man die von DE VRIEs et al. (1957) entwickelten Indikatorwerte für die einzelnen Pflanzenarten, die allerdings mehr Gültigkeit für Weiden als für Wiesen haben, auf die vorstehenden Ergebnisse der botanischen Untersuchungen anwenden würde, käme man sicherlich zu ähnlichen Abstufungen dieser Pflanzenbestände und von deren Standorten von trocken/frisch bis nass. Arten, die in den Glatthaferwiesen häufiger und anteilmässig stärker vertreten sind, haben $\mathrm{Vg}$ von bis $\mathrm{zu}-76$ (Arrhenatherum elatius), während Arten der feuchten und nassen Kohldistelwiesen $\mathrm{Vg}$ von über +80 bis über +90 aufweisen. Für die holländischen Grundwasserstandsversuche wurden mit Hilfe dieser Werte von Minderhoud (1960) und KRuINe (1960) relative Häufigkeitsziffern für den Feuchtegrad errechnet. Das bei dem Versuch in der Boker Heide zu tun, ist jedoch nicht möglich, da die meisten wichtigen Kennarten für bestimmte Standortseigenschaften bisher erst in Spuren, d.h. mit Anteilen von unter ein Prozent des Aufwuchses auftreten.

\section{LITERAT U R}

BOEKER, P.

\section{- u. H. G. Кмосн}

KLAPP, E.

Korsmo, E.

KRUIJNe, A. A.

en D. M. DE VRIES

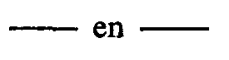

1959 Samenauflauf aus Mist und Erde von Triebwegen und Ruheplätzen. Z. f. Acker- u. Pflanzenbau. 108, 77-92.

1964 Die Verbreitung der wichtigsten Grünlandpflanzen NordrheinWestfalens in Abhängigkeit vom pH-Wert. Forsch, u. Beratung. Reihe B, Heft 10, 211-230.

1963 Grundwasserstandsversuche in der Boker Heide, Kreis Paderborn. Z. f. Kulturtechn. u. Flurbereinigung. 4, 142-156.

1949 Landwirtschaftliche Anwendungen der Pflanzensoziologie. Verlag E. Ulmer, Stuttgart.

1930 Unkräuter im Ackerbau der Neuzeit. Springer-Verlag, Berlin.

1960 Bruikbaarheid en betekenis van relatieve veelvuldigheidscijfers, indicatiegetallen en indicatiegraden in de graslandoecologie. Jaarb. I.B.S. 1960. 183-186.

1956 Gegevens betreffende belangrijke graslandplanten. C.I.L.O., Gest. Meded. No. 23.

1958 Een methode tot benadering van de voornaamste milieueigenschapppen van grasland aan de hand van de botanische samenstelling. Jaarb. I.B.S. 1958. 93-98. 
Minderhoud, J. W. 1960 Grasgroei en grondwaterstand. Ponsen en Looijen, Wageningen.

Tüxen, R., u. E. Preising 1951 Erfahrungsgrundlagen für die pflanzensoziologische Kartierung

VRIES, D. M. DE des westdeutschen Grünlandes. Angew. Pfl. soziologie. Heft 4.

1942 Over den invloed van jaargetijde en weer op de botanische samenstelling van grasland. Ned. Kruidk. Arch. 52, 303-307.

A. A. KRUUNe en H. MoOI

1957 Veelvuldigheid van graslandplanten en hun aanwijzing van milieu-eigenschappen. Jaarb. I.B.S. 1957. 183-191. 